\title{
EchoGéo
}

$13 \mid 2010$

Afrique, 50 ans d'indépendance : État et territoires

\section{Habiter un quartier RDP}

Processus de différentiation et d'appropriation socio-spatiale à Delft South au Cap

\section{Marine Suteau}

\section{OpenEdition}

\section{Journals}

Édition électronique

URL : https://journals.openedition.org/echogeo/11916

DOI : 10.4000/echogeo. 11916

ISSN : 1963-1197

Éditeur

Pôle de recherche pour l'organisation et la diffusion de l'information géographique (CNRS UMR 8586)

Référence électronique

Marine Suteau, « Habiter un quartier RDP », EchoGéo [En ligne], 13 | 2010, mis en ligne le 20 septembre 2010, consulté le 03 août 2021. URL : http://journals.openedition.org/echogeo/11916 ; DOI : https:// doi.org/10.4000/echogeo.11916

Ce document a été généré automatiquement le 3 août 2021

EchoGéo est mis à disposition selon les termes de la licence Creative Commons Attribution - Pas d'Utilisation Commerciale - Pas de Modification 4.0 International (CC BY-NC-ND) 


\section{Habiter un quartier RDP}

Processus de différentiation et d'appropriation socio-spatiale à Delft South au Cap

\section{Marine Suteau}

\section{NOTE DE L'AUTEUR}

Cet article est issu du mémoire de recherche de Master 1 de Géographie, Université Paris 1 Panthéon-Sorbonne, soutenu le 18/09/09 à l'Institut de Géographie de Paris 1, sous la direction de Myriam Houssay-Holzschuch. La recherche s'inscrit dans le programme Périsud (ANR nSUDS-07-046) dirigé par J.-L. Chaléard, chantier Le Cap. Le travail de terrain est soutenu par l'Institut Français d'Afrique du Sud (IFAS).

1 La question de l'évolution des villes sud-africaines constitue un sujet d'étude essentiel depuis la fin de l'apartheid alimentant une littérature fournie (Houssay-Holzschuch, 1999 ; Guillaume, 2001; Lemanski, 2001; Oldfield, 2000 ; Gervais-Lambony, 2003 ; Morange, 2006). L'héritage des pratiques de ségrégation s'observe aujourd'hui encore dans les structures des villes où les populations non-blanches ont longtemps été reléguées en périphérie dans des zones d'habitat homogènes racialement, les townships. Au Cap, ville fondée par les colons hollandais, les mouvements d'expulsions des populations non-blanches débutent en 1901 et sont systématisés au XXe siècle, avec la mise en place de la législation d'apartheid. Les plaines sableuses en périphérie du Cap (Cape Flats) sont alors investies pour reloger ces populations. À l'échelle nationale, le régime ségrégationniste d'apartheid intervient ensuite de manière systématique sur l'organisation des villes depuis 1950 avec l'application du Group AreasAct (Western, 1996). Suivant une logique de relogementdans les plaines sableuses tout au long du XX siècle, les townships africains de Langa, Gugulethu et Nyanga sont construits, ainsi que Mitchell's Plain et Khayelitsha (illustration 1), respectivement classifiés coloured et africain $^{1}$ et qui représentent une zone de 800000 habitants (Census, 2001). 
Illustration 1 - Localisation des townships du Cap selon la classification « raciale » d'apartheid

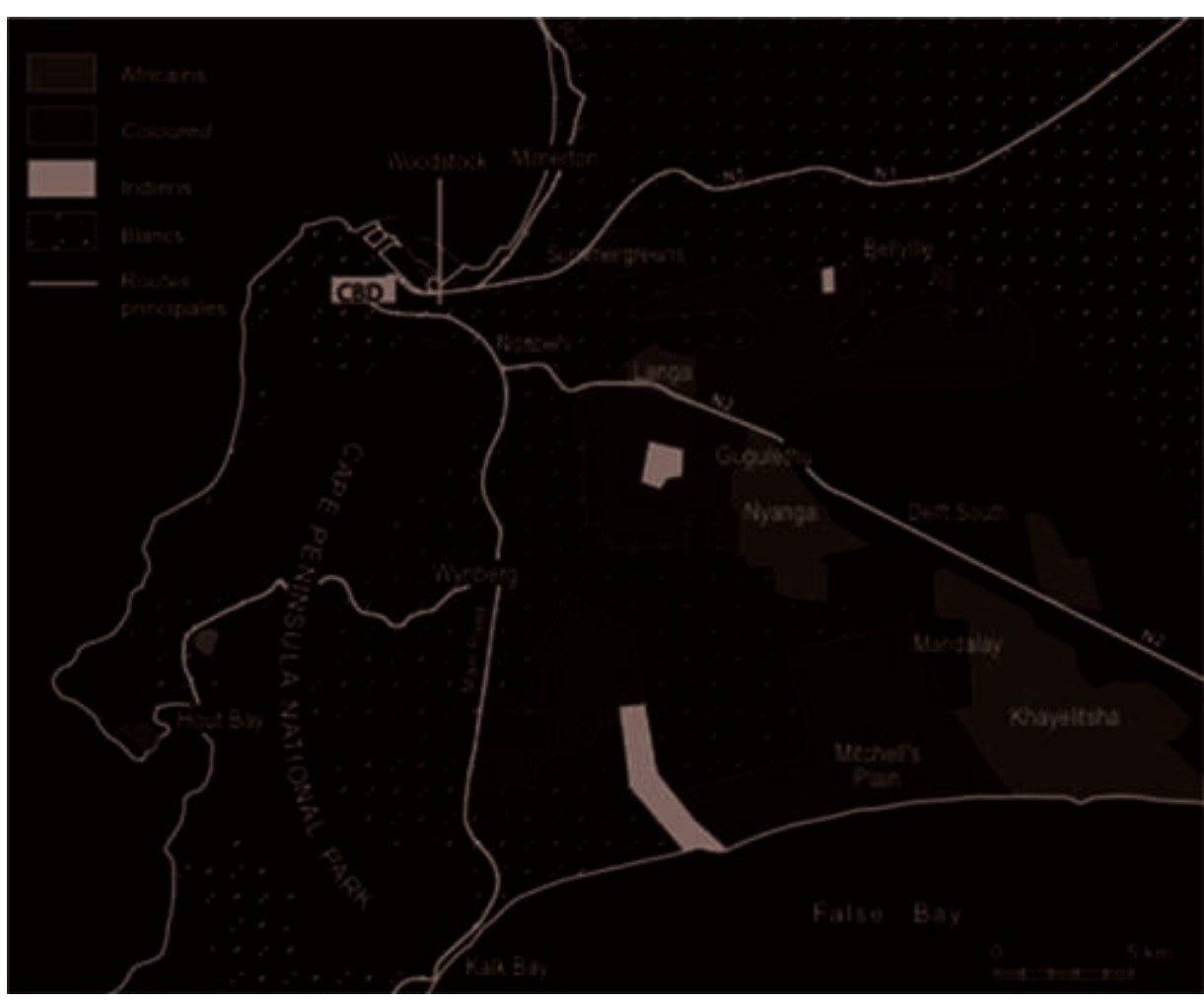

Source : Houssay-Holzschuch, 2001.

2 Suivant cette logique de construction de townships homogènes racialement dans les plaines sableuses, le quartier de Delft, construit pour les populations coloured, groupe « racial » le plus représenté au Cap, est réalisé dans la dernière décennie de l'apartheid. Les villes sud-africaines se sont développées en fonction de zones exclusivement réservées à un groupe de population sur le modèle de ségrégation résidentielle (Christopher, 2001). Le site de Delft, au nord de l'axe routier N2 (illustration 1), est hautement symbolique des virages idéologique et politique entrepris après les élections libres de 1994. Alors que l'apartheid prend fin, la question du logement se pose clairement pour le nouveau gouvernement de l'African National Congress (ANC) nouvellement arrivé au pouvoir. Il s'agira d'apporter des éléments de réponse, à partir d'un quartier post-apartheid, à une question qui polarise les disparités raciales et sociales héritées de l'apartheid.Les plans présidentiels, appartenant au programme de Reconstruction et de Développement (RDP), ont pour objectif de livrer des logements sociaux sous forme de maison standard qui, selon l'ascension sociale de ses propriétaires, s'agrandira. Le concept de la maison de départ (starter house) implique un système d'attribution spécifique selon des critères de revenu et une généralisation de la propriété privée. Les politiques de logements sociaux prévoient au départ un accès gratuit à la propriété privée et une close d'interdiction de revente. L'accession à la propriété privée devient le fondement de la reconstruction post-apartheid (Morange, 2006). Impulsés par les programmes présidentiels issus du RDP, de vastes projets de logements sociaux sont lancés dans les grandes villes. Les projets de maison standard de Devland à Johannesburg (Guillaume, 2001) et de Delft South au Cap (illustration 2) notamment, sont rapidement exécutés. Dès 1995-1996, des maisons sont construites sur le modèle d'une pièce unique, raccordée à l'eau et à l'électricité qui peut être agrandie à 
l'arrière de la parcelle par ses occupants. Au Cap, le projet d'aménagement résidentiel de Delft South est intégré au vaste plan de réhabilitation urbaine integratedServiced Land Project (iSLP) lancé par les autorités provinciales du Cap de l'Ouest (PAWC) (iSLP, 1996).

Illustration 2 - Maison RDP

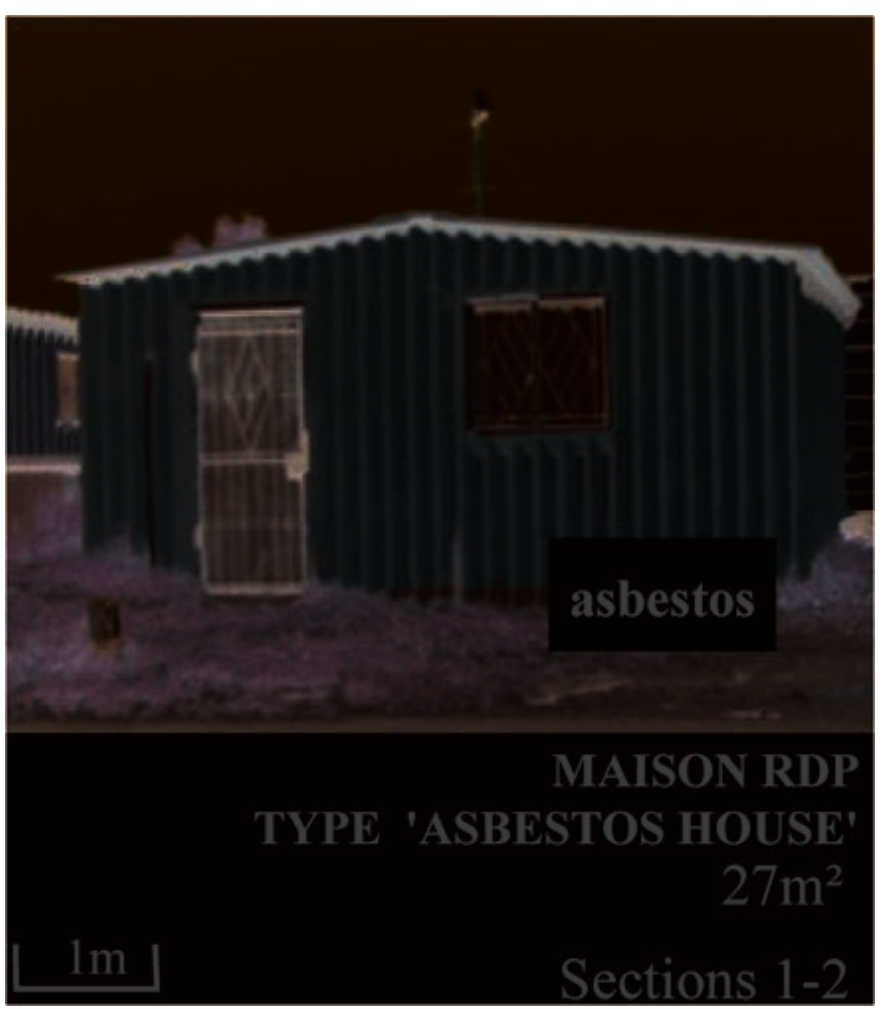

Auteur : Marine Suteau, 2009.

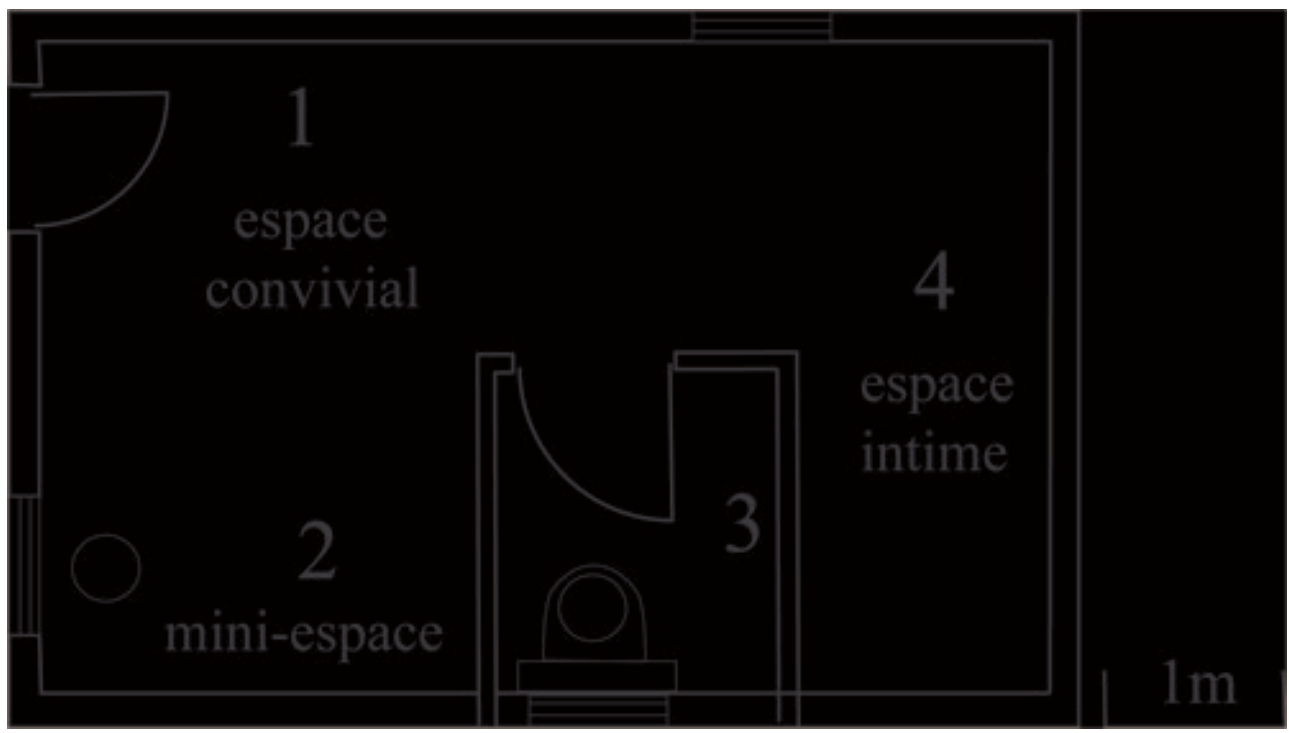

Source : iSLP, 1996. 


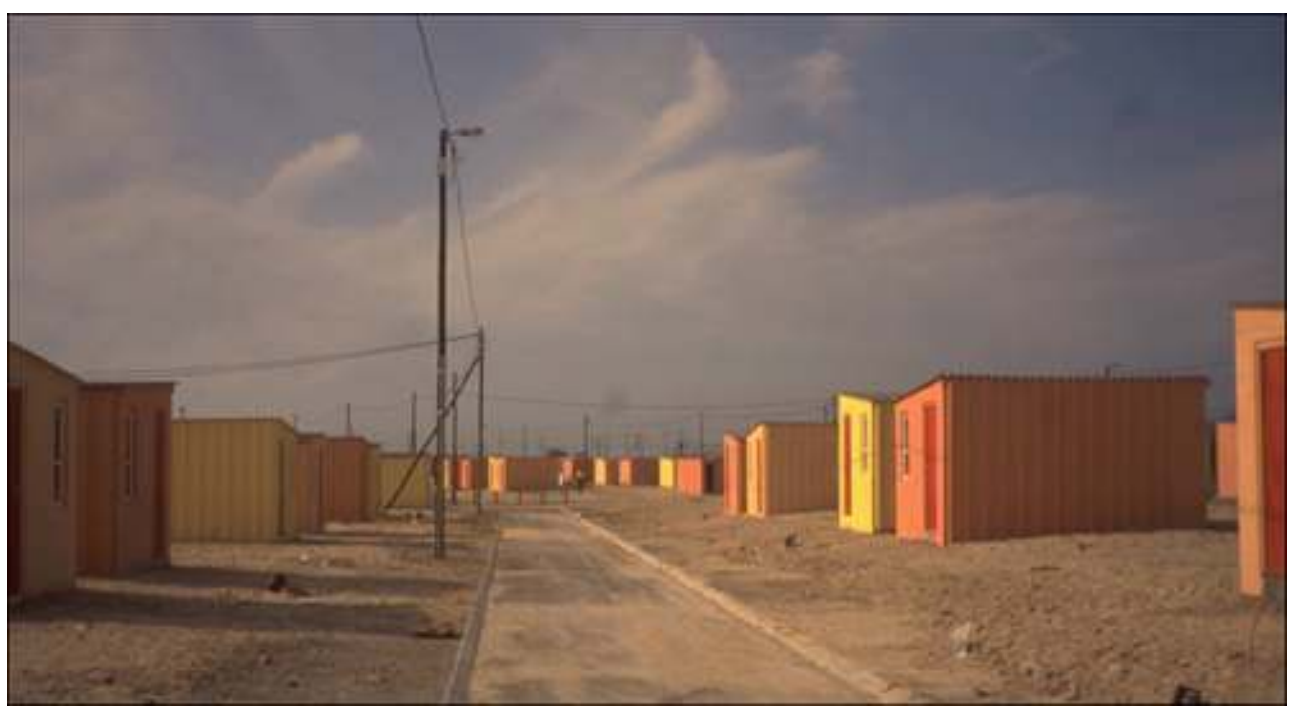

Auteur: Myriam Houssay-Holzschuch, 1997.

Habiter un quartier RDP au Cap devient une réalité dès fin 1996 pour des populations d'origine africaine et coloured, sur la base de critères de revenu. D'ailleurs, les premières enquêtes de terrain menées en 1996-1997 à l'installation des populations soulignent le caractère novateur des procédures d'attribution (Houssay-Holzschuch, 1999). Elles montrent des dynamiques communautaires nouvelles puisqu'un accord a été conclu entre l'administration provinciale chargée du programme ISLP et les représentants des populations coloured de Delft. Sur la base d'une parité $50 \%$ africain, $50 \%$ coloured dans l'attribution des logements, le nouveau quartier de Delft South se construit. Les autorités signent un changement radical avec la politique d'apartheid qui consistait à construire des logements de mauvaise qualité et surtout en sous-estimant les besoins en matière de logement pour les populations non-blanches (Houssay-Holzschuch, 1999). En conséquence, l'Afrique du Sud a entamé depuis la fin de l'apartheid des politiques de logements sociaux avec accès à la propriété pour réduire le retard en matière de logement qui affecte en premier lieu les populations des townships.

Les photographies (illustrations 3 et 4) présentent le quartier à 12 ans d'intervalle. Les baraques de tôles ondulées isolées autour des rues se sont transformées en pavillon individuel délimité par des clôtures, des buissons et des portails montrant l'appropriation du quartier par ses résidants. Delft South est ainsi révélateur de dynamiques d'appropriation et de différentiations socio-spatiales au sein d'un quartier de logement social mixte post-apartheid. 


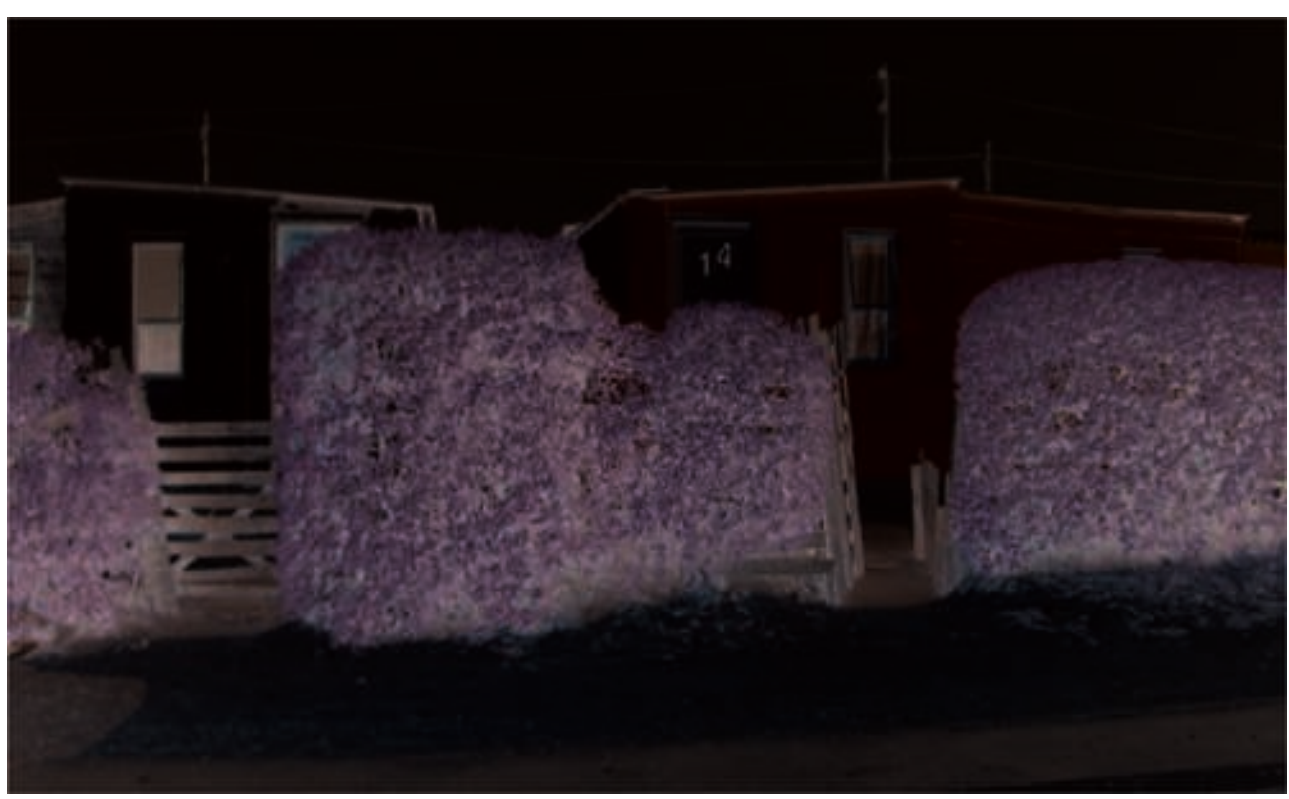

Auteur : Marine Suteau, enquêtes de terrain, 2009.

5 Cet article est étayé par quatre séries d'enquêtes (Houssay- Holzschuch, 1997 ; Oldfield, 2000 ; Gabriels, 2002 ; Millstein, 2008) et des articles de journaux locaux (Cape Argus, Cape Times) et nationaux (Mail \& Guardian); les enquêtes qualitatives ont été conduites de janvier à mars 2009 sous forme de 40 questionnaires remplis chez les résidants de Delft South, dans leur maison, en anglais. A l'échelle d'un quartier pauvre, périphérique et habité par des populations coloured et africaine, l'appropriation spatiale des maisons constitue un angle de différentiation sociale bien plus que "raciale» au sein de la population mixte de Delft South. La question de l'appropriation d'un quartier par ses habitants est posée, en analysant les évolutions socio-spatiales d'une politique publique de logements conduite par les autorités provinciales. Privilégiant l'approche " par le bas ", " au sens des citadins ordinaires plus ou moins organisés en collectif ou en associations » (Gervais-Lambony, 2003, p. 47), il s'agit de montrer en quoi l'action collective des résidants est emblématique des mouvements sociaux post-apartheid et des pratiques de différentiations socio-spatiales comparables à l'évolution générale de la société sud-africaine (sécurisation, privatisation des espaces, polarisation sociale etc.). En résulte une organisation du quartier selon des "communautés de rues", véritables groupes de quartier qui intègrent leurs membres et excluent les autres résidants.

\section{L'appropriation des maisons à Delft South : qui sont les protagonistes et comment l'action collective conteste les politiques publiques de logements sociaux?}

6 Les premières années à Delft South ont été rythmées par des mobilisations sociales, à l'origine de solidarités inter raciales et d'appropriation du quartier par les résidants sous forme d'actions collectives. Les conflits sociaux ont ponctué l'installation des 
populations à Delft South, phénomène illustré par les Door Kickers² (Oldfield, 2000) et par les illégaux de la Symphonie Way ${ }^{3}$ (Cape Argus, 15/03/09) entraînant la construction d'une zone de relogement provisoire. Ces différentes périodes de mobilisations révèlent des modes d'appropriation nouveaux : collectifs et « racialement » mixtes.

\section{Les protagonistes de Delft South : mise en place d'une gouvernance verticale}

7 La planification du projet résidentiel de Delft South participe à désengorger les listes d'attente pour le logement, autrefois divisées selon la catégorie « raciale », et à installer en périphérie du Cap des populations retenues par le programme iSLP. Les autorités provinciales sont avant tout les relais de la politique nationale de reconstruction incarnée par le programme RDP. Les procédures d'attribution des logements à Delft South sont significatives des changements introduits par la démocratisation du régime sud-africain en 1994, en ce qu'elles correspondent à un consensus entre les autorités provinciales (PAWC) en charge de la réalisation des maisons, les représentants coloured locaux de Delft, déjà présents, et les populations sélectionnées dans les townships africains du Cap. Delft South est construit au sud du quartier traditionnellement coloured de Delft, à l'est de l'aéroport. La section de Delft South construite de 1996 à 1999 représente à ce jour la plus importante section mixte coloured/africain du quartier en terme d'habitant.

Tableau 1 - Populations dans les sections du quartier de Delft en 2001

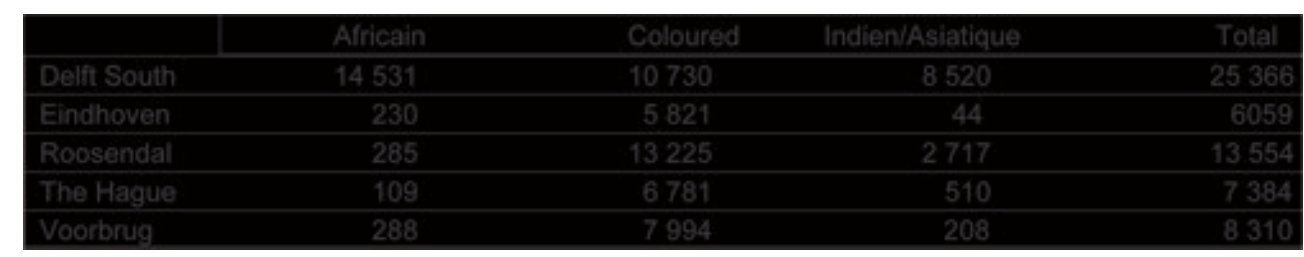

Source: Statistics SA, 2001.

Les sections Voorbrug, The Hague, Roosendal et Eindhoven correspondent aux zones de population coloured tracées sous l'apartheid. Delft South est la seule section à comptabiliser des populations africaines en 2001, qui y sont même majoritaires à $57 \%$. Les projets post-apartheid de logements sociaux qui succèdent au projet de Delft South (Delft Leiden, Delft N2 Gateway) sont eux-aussi réalisés sur la base d'une mixité « raciale », s'inspirant de l'exemple réussi de Delft South. 


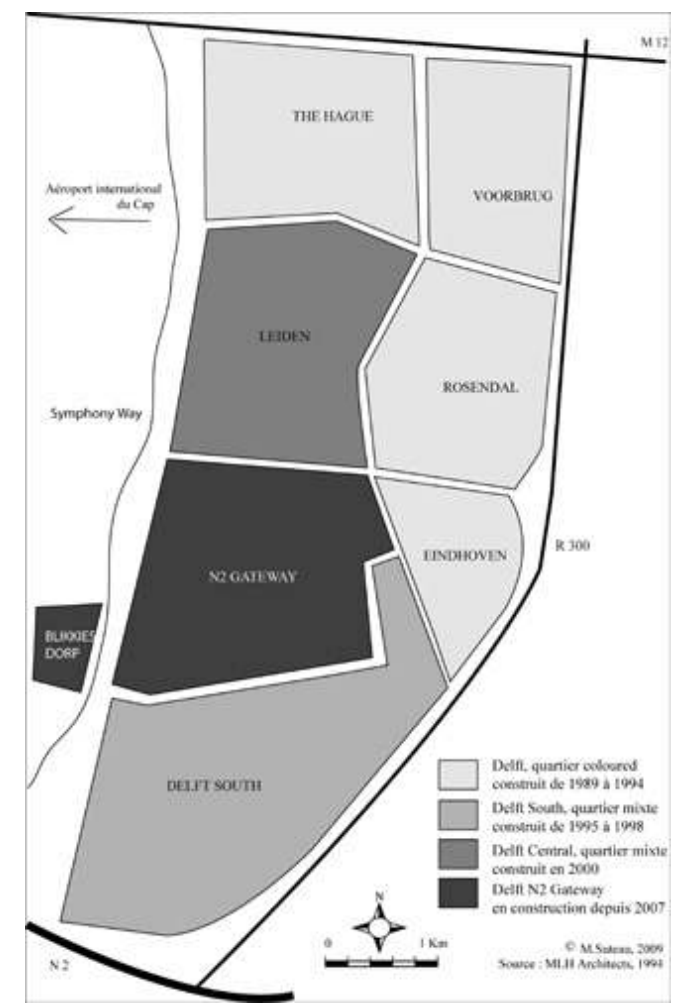

Réalisation : Marine Suteau, 2009. Source : MLH Architects, 1994.

9 Les projets résidentiels de Delft South, de Delft Central et de Delft N2 Gateway sont menés par les autorités provinciales depuis 1994, qui exécutent d'une part des politiques publiques de logement et intègrent d'autre part des acteurs locaux dans les réalisations. Les entreprises privées Thubelisha Homes sont notamment mandatées à Delft pour réaliser les logements sociaux (Smit, 2009). La mise en place d'une gouvernance verticale ouvre la voie à une contestation des résidants qui s'organisent en action collective.

\section{Gouvernance verticale et effets à l'échelle du quartier : les mouvements sociaux locaux contre les politiques publiques de logement}

Delft South est réalisé par les autorités provinciales qui, dans un premier temps, obtiennent un accord avec les élus locaux en 1996. Cependant, les occupations illégales des maisons en cours de construction par des populations en attente de logement sont récurrentes à Delft South: dès 1997, un groupe de résidants coloured a bloqué le chantier des premières sections (Cape Argus, 02/11/1997). Ces types de mobilisations sont représentatifs des modes d'action collectifs post-apartheid. L'appropriation collective et mixte pour un enjeu ponctuel, toujours en lien avec l'acquisition d'un logement, révèle des associations au-delà des solidarités « raciales » traditionnelles, qui sont très présentes dans les townships. La contestation des listes d'attribution des logements ${ }^{4}$ est un sujet brûlant qui rassemble tous les demandeurs de logements sociaux. La seconde vague de mobilisation marque un tournant dans les conflits sociaux à travers son caractère multi « racial ». Les enquêtes approfondies sur la période 1999-2001 (Oldfield, 
2001) ont montré l'émergence de solidarités nouvelles qui fonctionnent au-delà des solidarités « raciales ». Près de 1800 familles coloured et africaines se sont associées en 1998 avec la cellule locale de l'organisation South African National Civic Organisation (SANCO) pour s'emparer et occuper physiquement les maisons en cours d'attribution. L'organisation, créée en 1992, a pour objectif de faire participer les citoyens sudafricains à la jeune démocratie, notamment à travers des ramifications locales dans les townships. Les formes d'occupation illégale se caractérisent par le soutien de SANCO, reconnue pour ses actions de coopération entre les résidants locaux et le gouvernement post-apartheid. Ces populations coloured et africaines se sont présentées comme les Door Kickers, ce qui se traduit littéralement par ceux qui envahissent des maisons en défonçant leurs portes à coups de pied. Ainsi, comme le note S. Oldfield :

The 'Door Kickers', as they are known in the community, rejected the waiting lists and the system of housing allocation by claiming and then invading homes ${ }^{5}$.

En généralisant cette pratique d'occupation illégale, le phénomène devient médiatisé, et organisé, ce qui renforce l'efficacité de l'action collective. Les motivations des Door Kickers se justifient par un sentiment d'injustice vis à vis des listes d'attente, dénonçant manipulation et corruption. Depuis les années 2000, l'organisation SANCO, avec un réseau dense et des ramifications locales, a perdu de son influence dans les conflits qui succèdent au mouvement des Door Kickers.

La perte d'influence de SANCO et les scandales politiques de corruption ont pour conséquence de radicaliser les revendications sociales pour l'accès au logement sur le site de Delft. Le projet N2 Gateway (Smit, 2009) qui poursuit depuis 2002 les efforts de rattrapage en matière de construction de logements sociaux au Cap, se consacre à la réhabilitation des townships le long de la route N2 et à la construction de logements sociaux plus grands. Les critères d'attribution évoluent en proposant des logements plus variés, plus grands et subventionnés en fonction des tranches de revenus revues à la hausse. Le modèle de logement Breaking New Ground (BNG), aux standards de construction plus élevés pour des revenus supérieurs à 1500 Rands (soit 115 euros en 2009) remplace la maison RDP. A Delft, le projet N2 Gateway a pourobjectif dereloger les camps de squatters de Joe Slovo dans le township noir de Langa, à $15 \mathrm{~km}$ de Delft et les squatters locaux, provenant de la Symphony Way (Delft Central - illustration 5). Les conflits sociaux qui accompagnent le projet résidentiel N2 Gateway se sont renouvelés d'un point de vue idéologique. En s'opposant frontalement aux politiques d'aménagement urbain, et de ce fait au gouvernement, les mouvements sociaux portés par l'association contre les expulsions Anti Eviction Campaign (AEC), illustrent les revendications sociales. Expulsées des maisons N2 Gateway en cours de construction, un groupe d'une centaine de familles inscrites sur les listes du logement mais non retenues par le projet N2 Gateway aménagent un camp de squatters en 2007 le long de la route Symphony Way. 
Illustration 6 - Camp de squatters de la Symphony Way

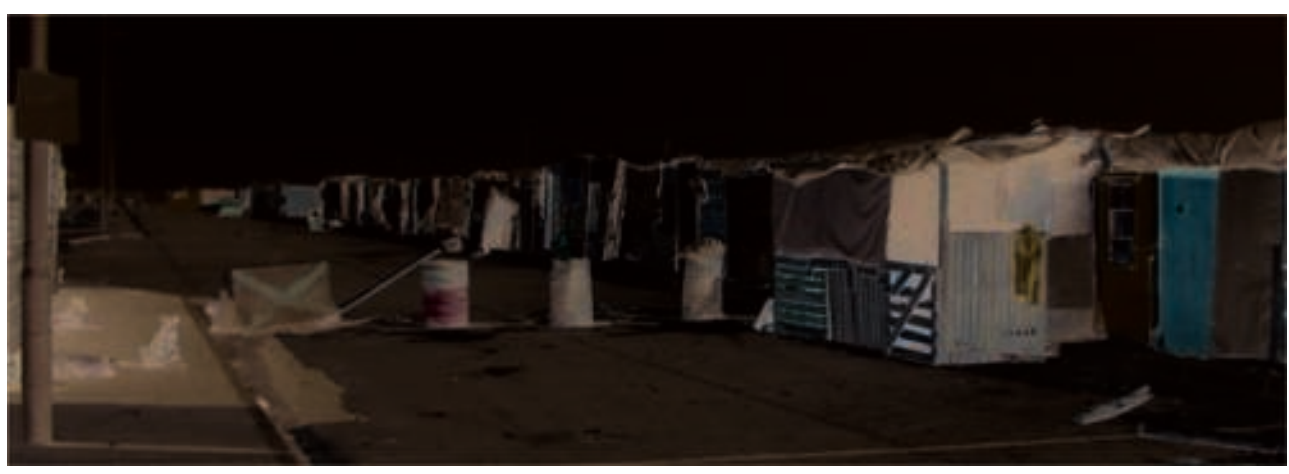

Auteur : Marine Suteau, enquêtes de terrain, 2009

Connues sous le nom des illégaux de la Symphony Way, les familles construisent des habitations précaires et occupent une portion de la Symphony Way qui est de fait fermée à la circulation (Ashraf Cassiem, 22/02/09). Soutenus par Ashraf Cassiem, représentant local de l'association AEC, ils dénoncent le caractère insuffisant du projet en matière de logements sociaux et les conséquences d'un habitat en périphérie. L'association AEC, mouvement central au Cap, conteste la politique du tout accès à la propriété privée qui mène à l'exclusion sociale. Les autres expulsés ont d'abord été conduits pour deux mois dans un campement de tentes pour être relogés dans une structure appelé Zone de relogement provisoire (TRA) à Blikkiesdorp ${ }^{6}$ (illustration 5). En 2008, sous le poids des demandes urgentes de relogement, les autorités provinciales et municipales ont commencé à construire un camp au nord de Delft (Smit, 2009).

Illustration 7 - Vues du Camp Provisoire de Blikkiesdorp (TRA)

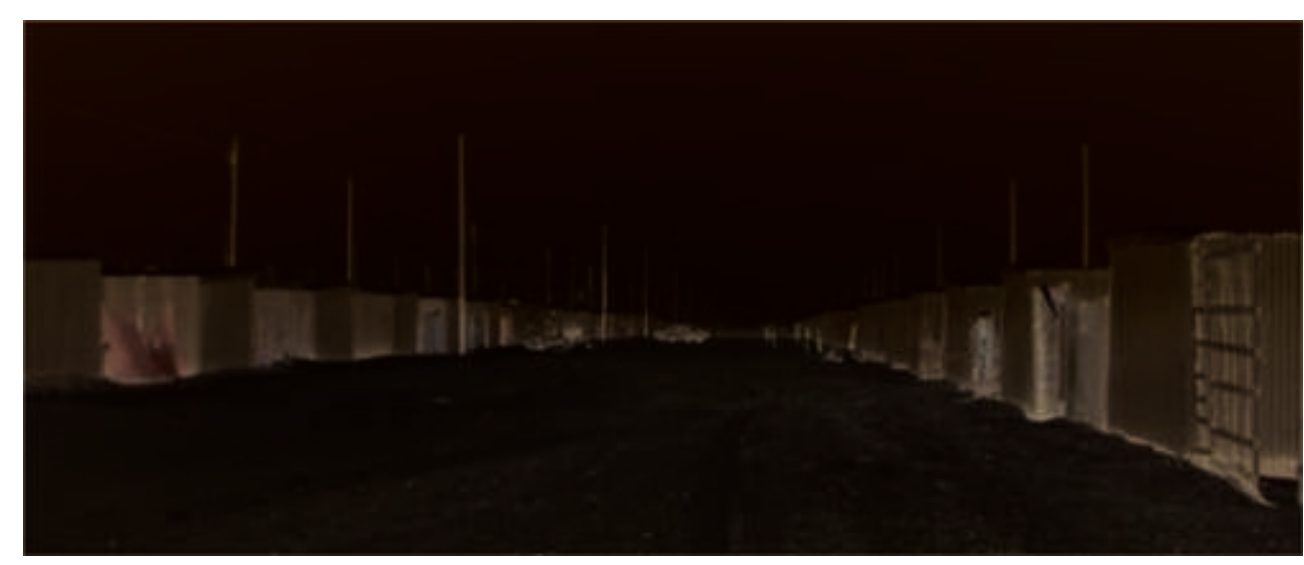

Auteur : Marine Suteau, enquêtes de terrain 2009.

Blikkiesdorp est un camp entouré de grillages surélevés de fils barbelés. Les maisons sont des baraques préfabriquées, en tôle ondulée, qui se divisent en deux sections. Le camp a été raccordé récemment à l'électricité, l'accès à l'eau reste limité en bordure des sections où des cabinets de toilettes mobiles sont installés. Cette forme de relogement correspond à la réalisation du programme de construction rapide des camps de relogements temporaires apparus dans l'urgence dès 2005 à Delft (Tsunami, zone de relogement bordant la Symphony Way) (DAG, 2005) (illustration 7). La construction d'un camp de relogement, avec des baraques mobiles qui comptent à ce jour 10000 résidants, soulève des questions structurelles en matière de gestion urbaine. 
S'agit-il d'une question de temps? Si oui, la résorption n'est pas immédiate mais progressive. D'autres logiques sont-elles à l'œuvre dans lesquelles la dynamique raciale est remplacée par une logique sociale et spatiale plus structurelle et plus insidieuse ? Malgré un recul de quinze ans sur les politiques publiques de logement au niveau national, les programmes de maison RDP sont toujours en cours de réalisation pour répondre à une demande de foyers en quête de stabilité résidentielle.

\section{Les dynamiques de normalisation du quartier : la mise en place de logiques sécuritaires}

Les anciens Door-Kickers, une fois installés légalement dans les maisons occupées, s'intègrent en adoptant des pratiques résidentielles similaires aux autres propriétaires $\mathrm{du}$ quartier, les mobilisations appartenant à la mémoire urbaine du quartier. Après les périodes de conflits liés à l'installation des populations, le quartier semble se construire d'après des logiques sécuritaires. Le mode d'organisation et de vie au quotidien se caractérise par des pratiques d'appropriation par agrandissement des maisons, révélant des stratégies de sécurisation. La stabilité résidentielle permet l'ascension sociale des résidants qui, selon les moyens financiers, aménagent leur espace en se protégeant. Les principales transformations du quartier correspondent à des logiques d'aménagement privées : par leur pratique d'agrandissement des maisons, les résidants poursuivent avant tout des logiques de sécurisation, voire de "fortification» de l'espace (illustration 8). Les éléments de protection comme les murets ou portiques sont peu à peu rénovés ou remplacés pour montrer le souci de chaque résidant en matière de sécurisation résidentielle, pratique d'ailleurs comparable aux quartiers de classes moyennes du Cap.

«On a décidé de se débarrasser du vieux portillon pour en mettre un plus grand, on n'a jamais eu de problème jusqu'à maintenant mais on voulait montrer que la maison est protégée .? $^{7}$

16 L'idée n'est pas tant de se protéger que de montrer qu'on se protège, ce qui valorise l'ascension sociale des résidants. 


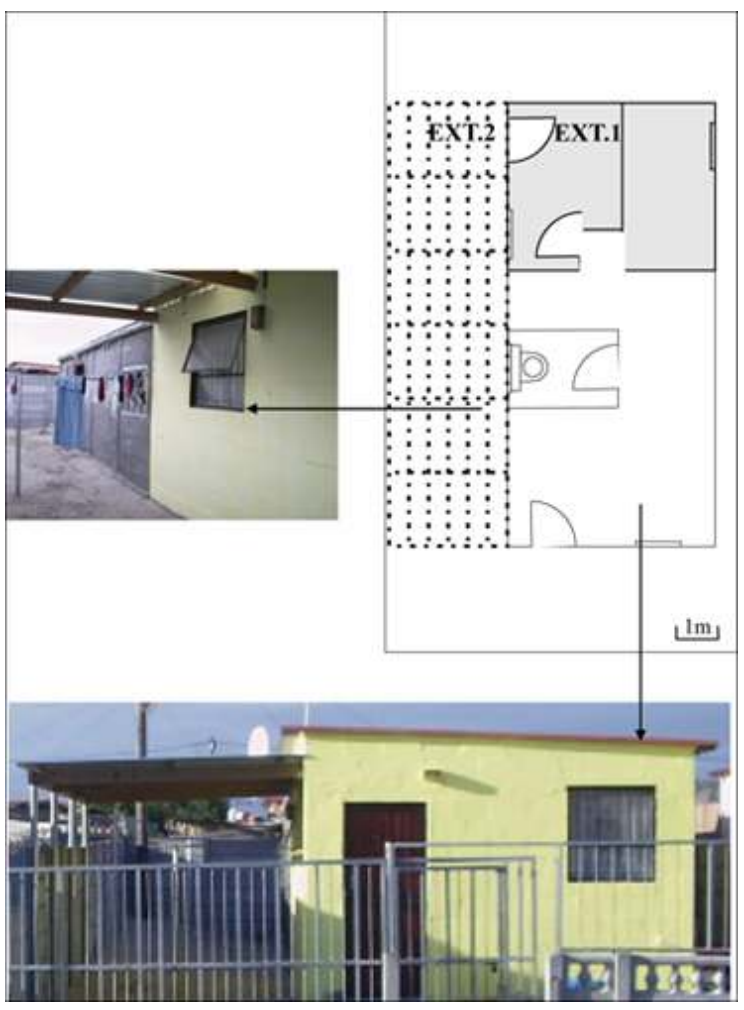

Auteur : Marine Suteau, enquêtes de terrain 2009.

17 Le quartier est considéré comme dangereux par les habitants qui se protègent en aménageant leur parcelle. Delft South est un espace de violences : les meurtres y sont en augmentation depuis les années 2000 (SAPS, 2009). Les autres formes de criminalité recensées sont majoritairement associées aux vols avec effraction suivis de violences physiques (cambriolage, 'car jacking', vol de voiture) si bien que les crimes contre les personnes et contre les biens sont intimement liés. L'appropriation des parcelles passe donc par leur sécurisation par les propriétaires. A ce titre, Delft South suit une trajectoire similaire à ce que l'on peut observer en matière de pratique de sécurisation des espaces privés dans les quartiers de classe moyenne au Cap. L'aménagement extérieur (fenêtres et porte renforcées, mur et portail de sécurité) reste la priorité à Delft South puisque plus des $3 / 4$ des enquêtés affirment sécuriser leur parcelle en priorité. Il s'agit d'une pratique collective sécuritaire, à l'initiative des propriétaires dont la nécessité est confirmée par les statistiques de criminalité. Ces pratiques de sécurisation se généralisent selon les moyens des ménages pour des raisons de sécurité et pour ne pas se situer en décalage vis-à-vis des maisons voisines.

Les programmes RDP avaient inclus dans le modèle de maison standard la possibilité d'agrandir la maison dans la parcelle (iSLP, 1996). Les questionnaires d'enquêtes révèlent une majorité de maison agrandie. Les extensions représentent un aménagement significatif puisque 32/40 personnes y font référence (enquêtes de terrain, 2009). L'aménagement par extension résulte en premier lieu de l'amélioration des conditions de vie pour les propriétaires de parcelle. Certaines maisons ne sont pas étendues, par manque de moyens financiers (4/40) ou par manque d'espace lorsque le jardin est sous-loué (15/40). Il s'agit d'une autre forme d'aménagement qui consiste à utiliser le jardin pour construire des structures informelles indépendantes. Ainsi se 
succèdent dans un même bloc de quartier des logements agrandis, des maisons mal entretenues et des baraques sous-louées à l'intérieur des parcelles. Ces types résidentiels révèlent une différentiation paysagère et sociale, à travers l'appropriation individuelle des maisons (extension, reconstruction,...). Les différents types d'aménagement et d'extension résultent de phases de construction en rapport avec les finances $d u$ foyer. Une première étape d'extension consiste à construire le nouvel espace en bois (EXT1, illustration 9). L'extension la plus fréquente est une extension de la longueur de la maison dans l'espace disponible à l'arrière de la parcelle. Le modèle de maison "standard" est un espace de promiscuité. Agrandir sa maison à l'arrière constitue une opportunité de construire de nouvelles chambres pour des foyers principalement intergénérationnels.

« J'ai décidé d'abattre les murs pour agrandir la maison à l'arrière. J'ai construit une chambre et une salle de bain en plus $» .8$

Puis, si les finances le permettent, l'extension se durcifie, comme en témoigne la présence de briques bâchées sur le côté gauche de la maison (illustration 9).

Illustration 9 - Maison étendue à l'arrière en cours de " durcification »

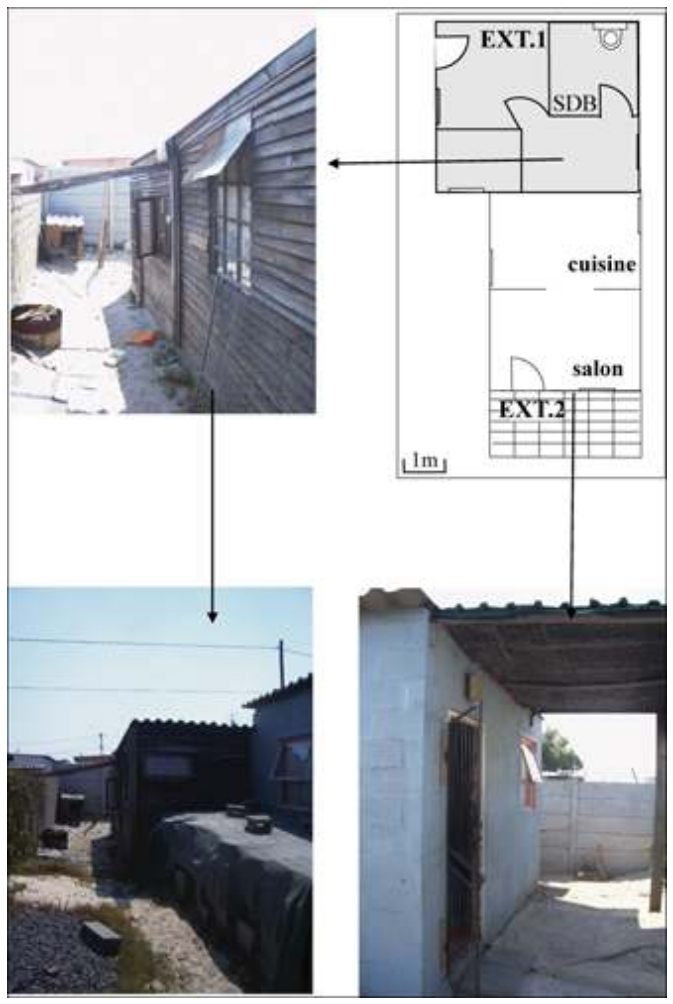

Auteur : Marine Suteau, enquêtes de terrain, 2009.

\section{Les « communautés de rues » : l'émergence de nouveaux pauvres en ville}

\section{Qui sont ces nouveaux pauvres?}

Selon les statistiques, l'essentiel des foyers de Delft South ne bénéficie pas de revenu fixe (Census, 2001). Il s'agit pour la plupart de travaux informels de survie, journaliers, 
notamment dans le bâtiment et la vente ponctuelle de marchandises périssables (enquêtes de terrain, 2009). Ce constat est tout à fait en adéquation avec les principes d'allocation du programme RDP puisqu'il privilégiait les revenus mensuels inférieurs à 1500 Rands. A contrario, les personnes ayant plus de 3000 Rands de revenus (soit 230 euros en 2009) appartiennent à la classe oubliée du programme RDP, considérées comme pas suffisamment pauvre pour être aidée (Morange, 2006). Le système de logements sociaux type maison RDP sur la base de la gratuité à l'accès pour les foyers sud-africains très modestes (revenus inférieurs à 1500 Rands) a évolué depuis 2002 vers un système davantage mixte, pour soutenir des foyers plus favorisés (revenus supérieurs à 3000 Rands. Cette couche sociale ne représente que $2 \%$ des habitants de Delft South. Cependant des tendances se distinguent entre ceux qui ont reçu gratuitement la maison RDP et ceux qui s'installent dans une maison par transaction financière. On peut émettre l'hypothèse que les nouveaux acheteurs sont ceux qui, en maintenant un revenu même modeste, construisent des parcours résidentiels au Cap, fondés sur des critères sociaux bien plus que " raciaux ». Ils se caractérisent par leur âge, entre 30 et 45 ans, par un foyer constitué de deux parents et de trois enfants en moyenne, et par la capacité à obtenir et préserver un emploi stable (6/40 propriétaires, enquêtes de terrain, 2009). Désireux de s'affranchir des codes familiaux et du contrôle social dont ils se plaignent, ils préfèrent s'offrir une indépendance résidentielle : les nouveaux propriétaires sont avant tout des ménages ayant connu une ascension sociale. En effet, les nouveaux arrivants interrogés conservent pour la plupart de la famille au Cap. Cependant, le partage des richesses s'effectue d'abord au profit du foyer. L'achat d'un logement social représente des stratégies résidentielles à l'échelle du ménage, ce qui illustre des mobilités résidentielles, conditionnées par le revenu, et des pratiques commerciales (transactions immobilières) en dehors des réseaux classiques « raciaux». L'achat des parcelles traduit des parcours de progression sociale qui se distinguent clairement de la majorité très pauvre à Delft South. A la lumière des enquêtes de terrain, $4 / 12$ familles de la rue enquêtée (illustration 10) ont des revenus supérieurs à 3000 rands par foyer, ce qui permet de dégager une tendance concernant les profils des propriétaires. Leur situation sociale leur permet de financer les aménagements de privatisation de l'espace privé et des ruelles adjacentes à l'intérieur des blocs de rues. À l'échelle du voisinage immédiat, ces pratiques de protection expliquent la différentiation spatiale constatée en matière de sécurisation. Par effet de propagation, ces aménagements dessinent des territoires qui se privatisent et se ferment à l'échelle des rues. Un nouveau résidant se souvient de ses conditions d'intégration à son arrivée :

« Je n'ai pas beaucoup de temps pour sympathiser avec les gens. Je ne vais pas au shebeen ${ }^{9}$. Je ne veux pas généraliser, les résidants ne vont pas tous au shebeen. Jeconnais bien mes voisins proches. A Khayelitsha, je connaissais tout le monde. Arrivé ici, je voulais vraiment apprendre à connaître mes voisins, ce qui est nécessaire pour rendre le quartier plus sûr. Avec mon travail en dehors de Delft, je ne sais pas les fréquentations de mes enfants. Je vouslais construite une environnement paisible et sécurisant dans ma rue $» .^{10}$

21 Les nouvelles pratiques d'intégration sont donc d'abord concentrées dans les espaces domestiques privés, sécurisant familles et biens matériels même modestes. Les formes de milices (groupes de vigilance) et les formes de replis sécuritaires sont le fondement des pratiques collectives. 


\section{"Les communautés de rues ", l'intégration par la mixité socio-« raciale»}

À l'échelle des rues s'organisent des solidarités collectives, vecteurs d'intégration spatiale. Les solidarités de rues, formes de "voisinage $»^{11}$, se manifestent d'abord à travers les relations sociales entretenues avec les populations voisines arrivées dans les mêmes périodes. Suivant les phases d'installation, les relations de voisinage garantissent une sécurité de chaque parcelle par la vigilance collective, notamment à travers l'identification visuelle. La protection des parcelles construit de fortes territorialités résidentielles (Dorier-Apprill, 2001) et la conscience d'appartenance à un groupe socio-spatial. Des voisins appartenant à une même rue s'organisent ensemble en s'appropriant non seulement l'espace privé de la maison mais aussi l'espace public de la rue adjacente.

«On a toujours gardé de bonnes relations de voisinage, les enfants peuvent jouer dans la rue et on surveille tous un peu. Il y avait un garçon qui venait d'une autre rue, suspecté de porter un couteau par les voisins. Ma femme a décidé de réunir les voisins et de les avertir que le garçon était dangereux ${ }^{12}$

Par crainte d'une intrusion, les membres de la communauté de rue exercent un contrôle social dans la rue, quitte à en limiter l'accès.

L'illustration 10 expose une communauté de rue et sa structure résidentielle. Les deux rues présentées illustrent la diversité des profils de populations à Delft South. On observe de fortes concentrations de populations coloured et africaines dans un espace limité. Plusieurs maisons d'une superficie de $32 \mathrm{~m}^{2}$ abritent de 4 à 6 personnes et quatre parcelles présentent des structures informelles sous-louées. L'espace privé est essentiellement résidentiel, seules deux parcelles sont exploitées commercialement en partie ou en totalité. La configuration des communautés raciales et leur date d'installation témoignent de recompositions raciales du quartier qui soutiennent une mixité africain/coloured. A l'échelle des rues, la coexistence de plusieurs groupes de population aux statuts fonciers différents, aux ressources financières variant de 0 à 13000 Rands par mois dont 3 foyers sans revenu fixe et à l'inverse 8 foyers avec deux revenus fixes (enquêtes de terrain, 2009) ne permet pas l'imposition d'un groupe socio-« racial ». En conséquence, les résidants ne peuvent pas revendiquer un territoire africain, coloured, de gang ou d'une classe sociale favorisée. C'est à travers la diversification des profils de populations que l'intégration est réussie à Delft South. Isaac $\mathrm{N}$. est un acteur moteur de la communauté de rues. Pendant la durée des enquêtes en 2009, il n'a cessé d'être sollicité par ses voisins pour régler toute sorte de conflits (domestiques, litiges vicinaux,...). Refusant toute étiquette politique, il participe néanmoins à de nombreux débats sur son quartier. 
Isaac N., d'origine africaine, a émigré au Cap dans les années 1990 comme de nombreux sudafricains pour trouver un emploi. Originaire de Cofinwaba dans le Cap de l'Est, il a réussi à retrouver de la famille à Khayelitsha (township africain du Cap), ce qui lui a permis de louer un logement pour sa famille dès son arrivée. Père de famille, il décide de s'inscrire à des cours du soir, se spécialise pour trouver un emploi au département des archives de l'Université du Cap (UCT) pour enfin neuf ans après son arrivée au Cap, acheter une maison à Delft South avec les 15000 Rands (soit 1150 euros) épargnés. Travaillant au Cap, son salaire mensuel est de $\mathrm{R} 11000$. Le père a pu conclure l'achat d'une maison à l'amiable en sollicitant son réseau professionnel. La même offre à Khayelitsha, leur ancien lieu d'habitation, ne les intéressait pas. Depuis son emménagement en quartier RDP à Delft South, il a réussi à gagner la confiance de ses voisins, même d'origine coloured, pour impulser la dynamique d'une communauté de rues. Habitant avec sa famille et ses trois enfants scolarisés à Delft South, il bénéficie d'une reconnaissance de ces voisins qui lui ont confié le rôle d'intermédiaire avec la municipalité pour présenter les demandes d'enregistrement des nouveaux propriétaires au registre général du cadastre.

Source : enquêtes de terrain, 23/03/09

Les initiatives commerciales, les différents statuts d'occupation du sol et les changements induits par les transactions immobilières créent de nouvelles logiques d'appropriation de l'espace et renforcent les relations de voisinage au-delà des appartenances «raciales ». Les relations de voisinage sont d'autant plus développées qu'elles cristallisent des intérêts communs. La reconnaissance visuelle, l'entraide entre voisins et les expériences techniques pour les agrandissements déterminent des modalités de voisinage. Ces relations de voisinage sont respectées, reconnues et font office de comité de vigilance, hérité des pratiques dans les townships africains :

«Il existe des street commitees ${ }^{13}$ à Khayelitsha, certains membres informant les résidants sur l'actualité du quartier. Ils aident les gens du quartier en cas de problème, comme les violences domestiques ou criminelles. Ils organisent des réunions avec les résidants (abahlali). Abahlali ou mhalali au singulier est employé en xhosa pour signifier les résidants. Dans certains cas extrêmes, le street commitee peut rejeter celui qui est jugé comme étant un criminel. Ils réunissent un comité et décident de son sort. La raison première de l'existence de ces réunions est la lutte contre la criminalité $»{ }^{14}$

Les violences domestiques et les criminalités sont repérées par les comités de vigilance qui tentent des médiations ou excluent des résidants aux comportements criminels. Même s'il n'existe pas de hiérarchie officielle dans le cas des relations de voisinage observées à Delft South, Isaac N. fait partie des personnes reconnues, auxquelles on peut se référer en cas de problème. Il bénéficie d'un capital social et culturel suffisant pour être leader, travaillant au Cap la semaine et participant régulièrement à des visites entre voisins le week-end. 
Illustration 10 - Structure résidentielle d'une communauté de rue ${ }^{15}$

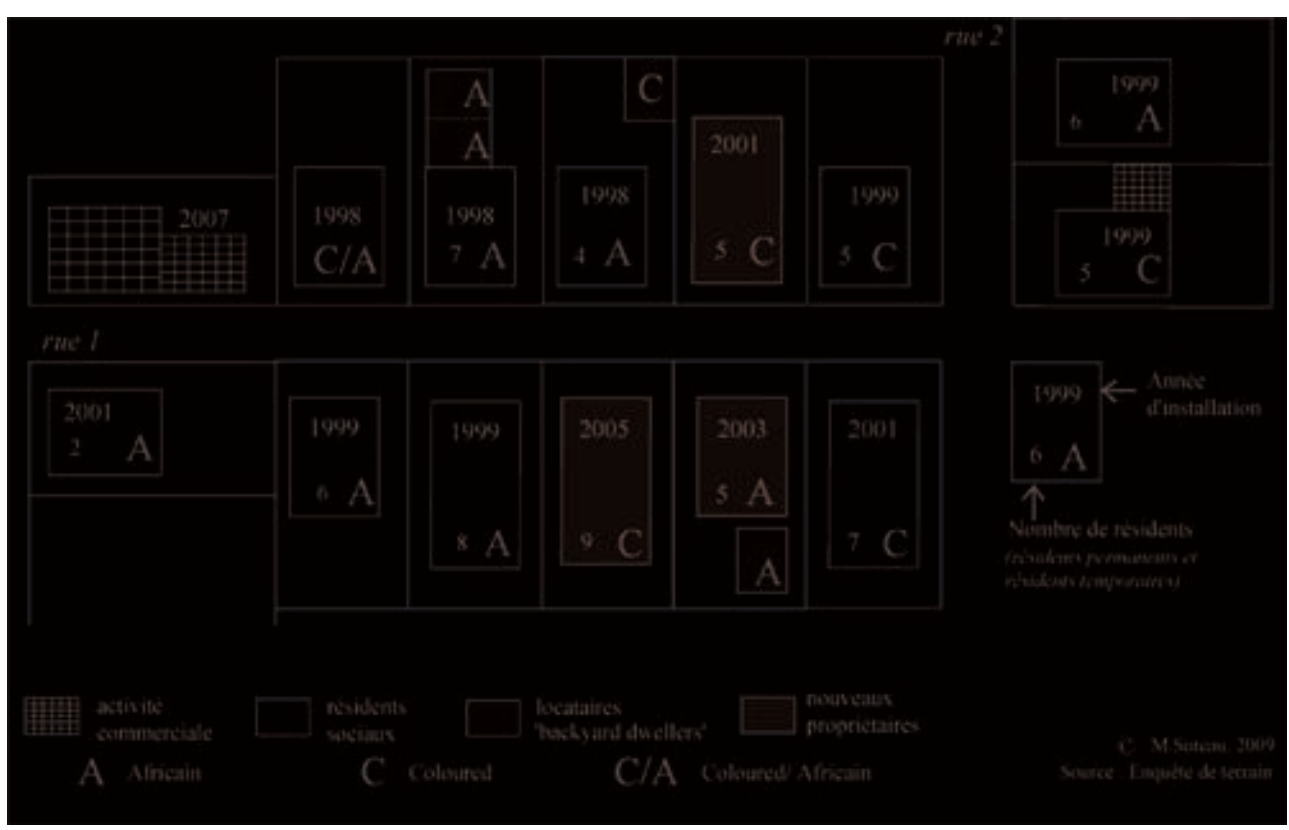

Echelle : $24 m^{2}$ - baraque témoin

Auteur: Marine Suteau, enquêtes de terrain 2009.

La communauté de rue a valeur de modèle d'intégration des résidants de la rue concernée, en mettant à l'écart les autres résidants. Au-delà de la simple illustration d'un groupement de voisin réussie, l'exemple est intéressant à plusieurs égards. Ces résidants privilégient l'espace privé qu'ils souhaitent sécuriser. Dans cette optique, ils préfèrent s'éloigner des bars (shebeens) pour se rapprocher des voisins et construire ensemble des relations sociales amicales. Par exemple, certains résidants n'hésitent pas à associer pratiques des bars et insécurité : "Je voulais créer un environnement paisible et sécurisant dans ma rue $»{ }^{16}$ D'ailleurs aucun bar clandestin n'a été relevé dans le voisinage immédiat. L'intégration des nouveaux arrivants ne se construit pas autour des lieux publics (superette, parking pour taxi collectifs, bibliothèque) qui sont des espaces de rencontre classiques, mais autour de la maison. Les relations amicales de voisinage peuvent s'approfondir en diversifiant les pratiques à l'échelle des rues. Les réalisations qui en résultent sont directement issues des résidants solidaires, organisés en groupe d'intérêts. Par exemple, la crèche vient soulager les habitants, notamment les femmes, qui ont l'opportunité de sortir du quartier pour travailler ou reprendre des études. Il y a ici des processus de différentiation socio-spatiale en cours selon les choix de la communauté de rues. Les crèches publiques restent trop peu nombreuses pour accueillir l'ensemble des enfants en bas âge à Delft South. La crèche photographiée cidessous (illustration 11) a été créée suite au vote d'une communauté de rues. Le choix des résidants, largement soutenu par les femmes de la communauté de rues, s'est porté sur l'aménagement d'espace de garde pour les enfants (vingt-trois enfants y sont à temps plein), ce qui résulte de l'évolution des besoins de la communauté et correspond à l'intérêt collectif/général. 
Illustration 11 - Crèche associative

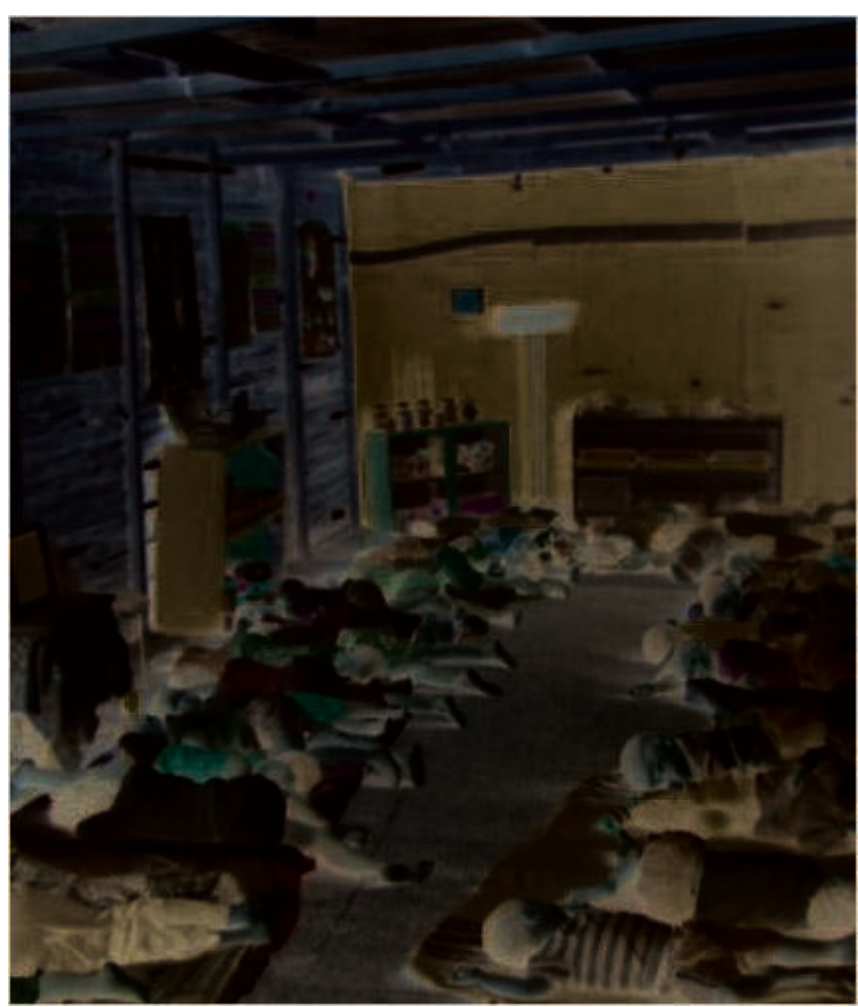

Auteur : Marine Suteau, enquêtes de terrain 2009.

28 L'espace de la crèche se situe à l'intérieur du territoire délimité par la communauté de rues, soit un total de 10 maisons (illustration 10). Le propriétaire a donné son accord pour transformer son jardin en activité commerciale, d'où l'extension en bois visible dans la pièce à jouer. Les employées, proches voisines de la maison-crèche, recrutées par la communauté de rues, contribuent au bon fonctionnement des lieux depuis deux ans. A ce titre, elles reçoivent un salaire versé par les parents, résidants de la communauté de rues. La crèche ne bénéficie pas de subvention de l'Etat et par conséquent, sélectionne d'abord les familles de la communauté de rues, quel que soit leur statut économique, social ou racial. Le tarif varie en fonction des ressources financières. Les prix s'échelonnent de 100 à 250 Rands par semaine par enfant. Le recrutement des enfants suit donc une logique communautaire dans le sens où les enfants de la communauté de rue sont retenus automatiquement et les autres enfants doivent être parrainés par un membre. La logique est d'abord de proximité. L'aspect sécuritaire, moteur des pratiques défensives et la sélection uniquement locale révèlent les limites du modèle d'intégration et on peut émettre des hypothèses concernant des dérives vers l'entre-soi, voire la «clubbisation». Face au succès rencontré, un agrandissement est envisagé, toujours en rapport avec l'intérêt collectif. Les candidatures sont parfois rejetées pour des raisons spatiales, ce qui témoigne des logiques d'exclusion dans le système de distinction entre ceux qui sont intégrés et en cours d'intégration (les nouveaux arrivants parrainés). 


\section{Conclusion}

Les maisons RDP de Delft South symbolisent le lancement des projets présidentiels postapartheid, la reconnaissance des populations non-blanches dans les villes, la possibilité de parcours sociaux ascendants mais aussi les espoirs contrariées de la jeune démocratie. La périphérisation continuelle des plus pauvres contribue à nuancer le caractère post-apartheid du Cap à travers le quartier mixte de Delft South. L'intégration urbaine des populations défavorisées sous l'apartheid est conditionnée au maintien - voire au développement d'une activité économique. Le chômage de masse (1/4 de la population, Stat SA, 2001) traduit aussi les inégalités "raciales » puisque seulement $4 \%$ des populations blanches sont affectées. Les populations défavorisées souffrent de la compétition pour le logement et se mobilisent pour occuper le champ social des revendications. Les pratiques déségrégatives observées sont donc limitées aux parcours ascendants des résidants. Les multiples formes d'appropriation des maisons illustrent la stabilisation résidentielle et familiale de sorte que le quartier résidentiel se transforme et s'organise en fonction de ses résidants. Les communautés de rues s'organisent sur le mode des solidarités de voisinage. La concentration d'intérêts détermine des projets collectifs et des projections spatiales au sein du territoire revendiqué, privatisé et protégé par la communauté de rues. La protection des biens et sécurité des personnes, motivée par les parcours ascendants, associe des propriétaires, des locataires et des populations au-delà des origines raciales. Ces modalités de voisinage se fondent sur des pratiques spatiales spécifiques -la sécurisation et privatisation des parcelles et des rues adjacentes-, ce qui participe à la production d'identités spatialisées - on est ainsi membre d'une communauté de rues. A l'inverse, la non-intégration à une communauté de rues accélère une polarisation sociale grandissante dans les périphéries résidentielles. Dans ce sens, les non-membres d'une communauté de rues sont les perdants des processus de différentiation et d'appropriation socio-spatiale du quartier.

\section{BIBLIOGRAPHIE}

Ashraf Cassiem, délégué local de l'association AEC, entretien, 22/02/09.

Christopher A.J., 2001. Atlas of a changing South Africa. London, Routledge, 206 p.

Beaud S., Weber F., 2003. Guide de l'enquête de terrain. Paris, La Découverte, 357 p.

Dorier-Apprill E., 2001. Le vocabulaire de la ville. Paris, Editions du Temps, 191 p.

Gabriels J., 2002. Low-cost housing in the Western Cape with special reference to Delft South and Wesbank. Bellville, Mémoire de Master (Géographie), University of Western Cape Press, $130 \mathrm{p}$.

Gervais-Lambony Ph., 2003. Territoires citadins, 4 villes africaines. Paris, Belin, collection

Mappemonde, $271 \mathrm{p}$. 
Gervais-Lambony Ph., Landy F. et Oldfield S., 2003. Espaces arc-en-ciel: identités et territoires en Afrique du Sud et en Inde. Paris, Karthala, 369 p.

Guillaume Ph.,2001. Johannesburg: Géographie de l'exclusion. Johannesburg/Paris, IFAS/Khartala, $392 \mathrm{p}$.

Houssay-Holzschuch M., 1999. Le Territoire volé. Paris, Thèse de Doctorat (Géographie), Université de Paris IV-Sorbonne, 287 p.

iSLP, 1996. Delft project committee. Cape Town, PAWC Publication, 1 p.

Lemanski C., 2006. The impact of residential desegregation on social integration: Evidence from a South African neighbourhood. Geoforum 37, 3.

Morange M., 2006. La question du logement à Port-Elizabeth. Paris, Karthala, 410 p.

Oldfield S., 2000. Urban networks, community organising and race. Minnesota, Thèse de Doctorat (Géographie), Université du Minnesota, 283 p.

Smit W., 2009. Le grand projet N2 Gateway. In Dubresson A., Jaglin S., 2009. Le Cap après l'apartheid, gouvernance métropolitaine et changement urbain.Paris, Khartala, p. 13-44.

South African Police Service, 2009. Comparative statistics. Cape Town, CIAC, 2 p.

Western J., 1996. Outcast Cape Town. Berkeley, University of California Press, 396 p.

\section{NOTES}

1. «Coloured» fait référence aux catégories raciales d'apartheid institutionnalisées par le Population Registration Act de 1950 (Gervais-Lambony, 2003). Dans l'aire métropolitaine du Cap, « coloured» qualifie le groupe dit «métis» traditionnellement de langue Afrikaans, « african », le groupe dit " africain » d'ethnie et de langue xhosa et " indien/asiatique », le troisième groupe classifié population non-blanches.

2. Oldfield S., 2000. Urban networks, community organising and race. Minnesota, Thèse de Doctorat (Géographie), Université du Minnesota.

3. Cape Argus ,15/03/2009. Symphony Way "illégals" take battle to court.

4. West N., 15/09/1996. Race row in Delft. Cape Town, Sunday Times Metro.

Norman J., 22/12/1998. Desperation makes Delft a housing hot potato. Cape Argus.

5. Oldfield, 2001, p. 174.

6. Mtyala Q., 27/10/200. Delft squatters shifted to Blikkies dorp. Cape Times.

Hartley A., 03/11/2009. Symphony Way families move to Blikkiesdorp. Cape Times.

7. Résidantes depuis 5 ans, 01/03/09.

8. Résidant depuis 6 ans, 14/02/09.

9. Les shebeens correspondent aux bars clandestins hérités de pratiques dans les townships pendant l'apartheid.

10. Homme d'origine africaine, propriétaire.

11. «On peut considérer le voisinage comme l'ensemble des relations sociales que les citadins développent sur fond de proximité résidentielle, avec les personnes qu'ils perçoivent comme leurs voisins au sens large » (Gervais-Lambony, 2003: 59).

12. Isaac N., 23/03/09.

13. Dans les townships africains, les comités de rue (street committees) fonctionnent sur le mode du partage d'intérêt depuis l'apartheid, reconnus comme représentants locaux des civics. L'objectif principal est de faciliter l'organisation des relations de voisinage malgré des conditions de vie précaires et de remplacer les services policiers peu fiables. Dans le cas des communautés 
de rues à Delft South, les relations de voisinage s'associent aux services de protection de la police pour combattre les territoires revendiqués par les gangs. Les comportements criminels font l'objet d'une surveillance de la part des membres de la communauté de rues qui n'hésitent pas à en informer les services de police.

14. Homme d'origine africaine, résidant depuis 8 ans à Delft South, 21/02/09.

15. La notion de communauté est ici entendue comme un groupe de voisins à partir duquel se crée un lien social, un sentiment d'appartenance et un phénomène d'identification.

16. Homme d'origine africaine, propriétaire.

\section{RÉSUMÉS}

Cet article porte sur les processus de différentiation et d'appropriation socio-spatiale dans un quartier d'habitat social issu des programmes de Reconstruction et de Développement (RDP) postapartheid. Situé dans lesplaines sableuses du Cap, le quartier périphérique de Delft South pose le débat de l'évolution des quartiers RDP dans les villes sud-africaines. Dans le contexte postapartheid, les stratégies d'accès à la propriété et les résistances locales participent à la production de territoires appropriés, différenciés et revendiqués. Les communautés de rues, fondées sur les solidarités mixtes de voisinage, illustrent les recompositions socio-spatiales en quartier RDP.

This article focuses on a socio-spatial analysis of territorial change in Cape Town over timewith the study of Delft South as a post-apartheid low-cost housing development program. It asks how successful or not RDP programs have been in South African cities. It argues that social differentiation is in process. In the post-apartheid, strategies to access housing and social movements are producing socio-spatial territories. Through housing and socio-spatial networks inherited from street committees, Delft South may constitute a complex peripheral space with the creation of specific racially mixed neighborhoods.

\section{INDEX}

Mots-clés : appropriation, communauté de rue, différentiation, maison RDP, quartier mixte

Keywords : low-cost housing, racially mixed neighborhoods, RDP house, social differentiation

\section{AUTEUR}

\section{MARINE SUTEAU}

Marine Suteau est actuellement étudiante en M2 à l'Université Paris 1 Panthéon-Sorbonne. Elle a soutenu en 2009 sous la direction de Myriam Houssay-Holzschuch un mémoire de master 1 : Recompositions urbaines post-apartheid. Le quartier d'habitat social de Delft South au Cap ; un film est en préparation en partenariat avec l'Université Paris Panthéon-Sorbonne, l'IRD audiovisuel et l'Institut Français d'Afrique du Sud : Blikkiesdorp, la vie en boîte. m.suteau@hotmail.fr 